\title{
Prospects of Building Capacitive Nonlinear Lines Using Ceramic PZT for High-Frequency Operation
}

\author{
Jose O. Rossi ${ }^{1}$, Lauro P. Silva Neto ${ }^{1}$, Fernanda S. Yamasaki ${ }^{1}$, and E. Schamiloglu ${ }^{2}$ \\ ${ }^{1}$ Associated Plasma Laboratory, INPE, Sao Jose dos Campos, SP 122227-010, Brazil \\ ${ }^{2}$ Dept. of Electrical and Computer Engineering, University of New Mexico, NM 87131-0001, USA
}

\begin{abstract}
The objective of this paper is the study of ceramic dielectrics for nonlinear transmission line (NLTL) applications in high-voltage (HV) compact pulsed power systems at high frequencies. Barium and strontium titanate (BST) ceramics have been used with great success as excellent dielectrics in the construction of high voltage (HV) commercial ceramic capacitors with reduced dimensions because of their high dielectric constant. However, the attempts to reach frequencies above 500 MHz up to $1 \mathrm{GHz}$ using BST ceramics in nonlinear lumped lines have been unsuccessful due to the loss in the dielectric material that limits the operating frequencies up to about $250 \mathrm{MHz}$. On the other hand, these results indicated that the use of a lower loss nonlinear dielectric of reduced permittivity could be the solution for achieving higher frequencies in nonlinear capacitive lines. Therefore, the main point of this work is to characterize another type of ceramic known as PZT (Lead Zirconate Titanate), which is promising for NLTL applications.

Index Terms - PZT ceramics, nonlinear transmission lines, $\mathrm{RF}$ generation, dielectrics
\end{abstract}

\section{INTRODUCTION}

The attempts to obtain oscillating pulses at frequencies above $500 \mathrm{MHz}$ up to $1 \mathrm{GHz}$ using ceramics based on barium/strontium titanate (BST) in nonlinear lumped lines have been unsuccessful due to the loss in the dielectric material that limits the operating frequencies below $100-250$ $\mathrm{MHz}[1,2]$. The analysis of these results also indicated that the use of a nonlinear dielectric with lower permittivity would be very good for achieving higher frequencies in nonlinear transmission lines made using dielectric materials, termed nonlinear capacitive lines (NLCLs). In the search for new dielectrics for these applications, PZT ceramics can be a good alternative because of their reduced dielectric constant (1000) compared to BSTs (on the order of 5000) as frequency depends on the inverse of $\sqrt{ }(\mu \times \varepsilon)$ and also because of lower losses (i.e. lower loss tangent). PZTs are also ceramic composites, but in this case made of Lead $(\mathrm{Pb})$, Zirconium (Zr) and Titanium (Ti), being basically used in industrial and applications as sensors, transducers, dynamic force/pressure meters and explosive detonators in ferroelectric generators, among others. Like BSTs, they have a Perovskite crystal structure on a tetragonal form below the Curie temperature $\left(T_{c}\right)$. When heated above $T_{c}$ they lose their piezoelectric properties (thermal de-poling) as the structure becomes symmetric and cubic, exhibiting no net dipoles (paraelectric phase). Unlike BSTs (with $\mathrm{T}_{\mathrm{c}} \approx 20^{\circ} \mathrm{C}$ ), they are in the ferroelectric phase at room temperature because of their elevated $\mathrm{T}_{\mathrm{c}} \approx 300^{\circ} \mathrm{C}$. In view of that, for use as dielectrics in NLCLs applications, PZT ceramics must be de-poled to remove their piezoelectric properties, avoiding in this way dielectric losses and damages caused by mechanic deformation on pulsed conditions.

As pulsed HV operation is involved in NLTL applications, the dielectric breakdown strength (BD) of these materials on pulsed condition is an important parameter to be measured [3]. Moreover, other important characteristic to be considered for use in NLTLs is the assessment of their nonlinearity [4], since for nonlinear applications polar dielectric materials (such as BST and BT ceramics) are normally employed because their dielectric constant varies with the applied voltage, temperature, frequency, etc. Therefore, the main objective of this paper is to show the characterization of PZT material in terms of their nonlinearity, losses and BD strength to assess their possible use in NLCLs at higher frequencies. For this, the acquired dielectric PZT samples were previously de-poled at the factory by a thermal process and characterized at our laboratory by measuring their capacitance as a function of the applied voltage $(\mathrm{C} \times \mathrm{V})$, temperature $(\mathrm{C} \times \mathrm{T})$ and frequency $(\mathrm{C} \times \mathrm{f})$. The linearity of the dielectric material and their losses were investigated by measuring the $\mathrm{P} \times \mathrm{E}$ polarization curve and loss tangent (or dissipation factor) with frequency, followed by the HV BD tests under single shot conditions as the samples are damaged after BD rupture. The main PZT results from these tests were also compared with BST dielectrics of commercial ceramic capacitors.

The next section of this paper shows the experimental setup employed to carry out the characterization tests of the dielectric. The main results obtained so far and discussions are given in the third section and the conclusions of this work and prospects for using PZTs in NLCL applications are presented in the final section. 


\section{EXPERIMENTAL SET-UP}

The $\mathrm{C} \times \mathrm{T}$ measurements were performed in the range of $20-200^{\circ} \mathrm{C}$ by placing the devices under test inside an oven with a controlled temperature ramp of $0.05^{\circ} \mathrm{C} / \mathrm{s}$. A capacitance meter, connected to the electrodes of the PZT or of the ceramic capacitor, and a thermocouple attached to the bulk of both were used to give the values of $\mathrm{C}$ and $\mathrm{T}$ during a run of the ramp (until the maximum temperature set at $200^{\circ} \mathrm{C}$ was achieved). As the sample dimensions are known the corresponding dielectric constant $\mathrm{k}$ was calculated and also plotted against $\mathrm{T}$. For measurements as a function of frequency, an HP 4285A/ $30 \mathrm{MHz}$ RLC impedance meter was used to measure the variation of the capacitance, equivalent series resistance and loss tangent with frequency for both devices. For $\mathrm{C} \times \mathrm{V}$ measurements a $10 \mathrm{kV}$ HV DC power supply was used to charge the device under test. A capacitance meter connected in series with the device and isolated from the HV DC power supply using a HV linear blocking capacitor of higher capacitance (at least $1.0 \mu \mathrm{F}$ ) measured the nonlinear capacitance under test. More details of the circuit can be found in [4]. With the $\mathrm{P} \times \mathrm{E}$ polarization measurement the well-known Sawyer-Tower circuit [5] was used. In this case, the PZT sample or the capacitor under test were fed by an $\mathrm{AC} 60 \mathrm{~Hz}$ step-up transformer ( $10 \mathrm{kV}$ maximum) in series with a HV linear capacitor of higher capacitance of $0.75 \mu \mathrm{F}$, where the transformer output voltage was controlled via an input autotransformer. In this measurement system, the voltage across the transformer output is proportional to the electric field applied in the dielectric whereas the voltage across the linear capacitor is proportional to the polarization $\mathrm{P}$ or the accumulated charged $\mathrm{q}$ ( since $\mathrm{P}=\mathrm{q} / \mathrm{A}$, where $\mathrm{A}$ is the area of the dielectric). Finally, for the HV BD tests an LC oscillatory half sine wave discharge circuit was built [4]. This circuit comprised basically a $1 \mathrm{kV}$ capacitor bank that is discharged via the closing of a mechanical normally open switch through the primaries of two $12 \mathrm{~V}$ ignition coils connected in parallel and in anti-phase in order to generate a maximum HV differential output pulse of about $60 \mathrm{kV}$ across the electrodes of the PZT sample or of the capacitor tested.

\section{RESULTS AND DISCUSSION}

A few tens of de-poled PZT type 4 and 8 samples were acquired from a Brazilian company (ATCP) with $30 \mathrm{~mm}$ and $25 \mathrm{~mm}$ diameters, respectively, and both of $2 \mathrm{~mm}$ thicknesses. PZT-4 and PZT-8 have basically the same electrical properties, but with slight differences in their composition to attain certain differences in piezoelectric properties. The samples have both (bottom and top) surfaces covered with metallic silver coated electrodes that gives capacitances in the range of $\mathrm{nF}$ (approximately $3 \mathrm{nF}$ for type 4 and $2 \mathrm{nF}$ for type 8). Figure $1 \mathrm{~b}$ shows the $\mathrm{C} \times \mathrm{T}$ characteristic in black for a type 4 PZT, where a nonlinear behavior can be seen clearly as a function of temperature since PZTs are ferroelectric material and present an elevated Curie temperature $T_{c}$. As expected below $\mathrm{T}_{\mathrm{c}}$ (from room temperature up to $200^{\circ} \mathrm{C}$ ) there is an increase in the dielectric constant in blue (or capacitance in black) from 1000 up to 3250, approximately. On the other hand, comparing this result with that measured from $-35^{\circ} \mathrm{C}$ to $200^{\circ} \mathrm{C}$ as shown in Figure $1 \mathrm{~b}$ for a commercial ceramic capacitor of $10 \mathrm{nF} / 2 \mathrm{kV}$ whose dielectric is a BST composite, ensures that the BST was in the paraelectric phase at room temperature $\left(\mathrm{T}_{\mathrm{A}}\right)$ as the dielectric constant of the capacitor decreased as expected from 12000 to 3000, approximately, at $200^{\circ} \mathrm{C}$. In both cases a nonlinear behavior was observed, but with increasing $\mathrm{C}$ (or k) for PZTs at higher $\mathrm{T}$ and the opposite, i.e. decreasing $\mathrm{C}$ (or $\mathrm{k}$ ) with $\mathrm{T}$ increase after $\mathrm{T}_{\mathrm{A}}$ for ceramic commercial capacitors. Note that for $\mathrm{T}<\mathrm{T}_{\mathrm{A}}$ the BST dielectric is in the ferroelectric phase as is the PZT [6].

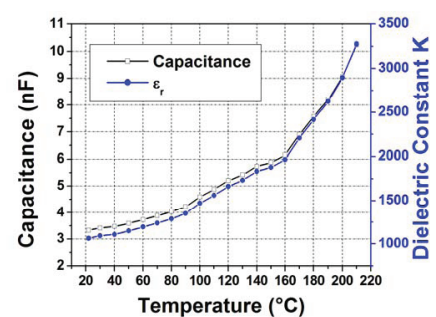

(a)

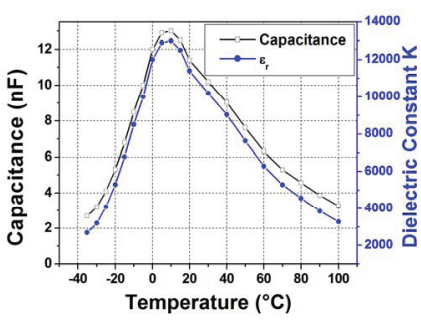

(b)
Figure 1. CxT measurement for : (a) the type 4 PZT sample and (b) 10 $\mathrm{nF} / 2 \mathrm{kV}$ ceramic capacitor.

For the $\mathrm{C} \times \mathrm{V}$ tests, a tiny surface area of the PZT sample near its border was removed and the PZT was immersed in oil at room temperature to avoid surface breakdown. Figures $2 \mathrm{a}$ and $2 \mathrm{~b}$ show the $\mathrm{C} \times \mathrm{V}$ curves (in black) obtained for the depoled PZT type 4 sample and the $0.47 \mathrm{nF}$ BST capacitor of 3 $\mathrm{kV}$ for comparison. For the PZT sample, its $\mathrm{C} \times \mathrm{V}$ characteristic is more linear, showing a variation of only $17 \%$ compared to the BST capacitor dielectric with corresponding variation of $50 \%$. Similar behavior was found for other PZT samples and capacitors tested. For PZT and capacitor, note that $\mathrm{k}$ decrease follows the capacitance variation as shown in Figures $2 \mathrm{a}$ and $2 \mathrm{~b}$ by the blue curves. In both cases, this decrease is explained by the alignment saturation of the electric dipoles as the electric field is increased [7].

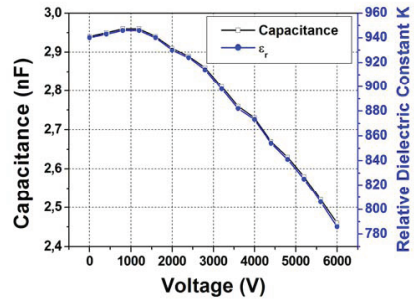

(a)

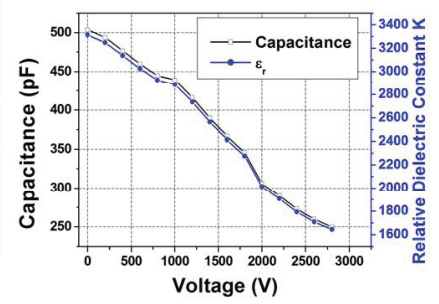

(b)
Figure 2. $\mathrm{C} \times \mathrm{V}$ measurements for: a) type 4 de-poled PZT sample and b) a ceramic capacitor of $0.47 \mathrm{nF} / 3 \mathrm{kV}$.

For the frequency measurements, the important parameters for characterization of dielectrics that were measured were: C, dissipation factor $(\tan \delta)$, impedance $(Z)$, phase angle $(\theta)$, and series equivalent resistance $\left(\mathrm{R}_{\mathrm{ES}}\right)$ [8]. As an example, Figure 3a shows $\mathrm{Z}$ (in blue line) and corresponding $\mathrm{R}_{\mathrm{ES}}$ (in black) in $\Omega$ from $100 \mathrm{kHz}$ to $30 \mathrm{MHz}$ for the $10 \mathrm{nF} / 2 \mathrm{kV}$ capacitor. Note that both $\mathrm{Z}$ and $\mathrm{R}_{\mathrm{ES}}$ decrease with frequency as expected 
since $X_{C}=1 /(\omega C)$ and the parallel dielectric loss resistance $R_{D}$ is reflected in series as $1 /\left(\omega^{2} C^{2} R_{D}\right)$. For $R_{E S}$ measurement, the small series resistance of the capacitor leads/plate foils (actual series resistance $-\mathrm{R}_{\mathrm{AS}}$ ) and the leakage current effect represented by a parallel resistance $R_{L}(G \Omega)$ are neglected. $\mathrm{R}_{\mathrm{AS}}$ is not negligible when the skin effect predominates at higher frequencies in excess of several $\mathrm{GHz}$ and $\mathrm{R}_{\mathrm{L}}$ is exceptionally low at very low frequencies. However, at a frequency on the order of $1 \mathrm{MHz}$ the inductance of the capacitor leads resonates with its capacitance and the inductance effect takes place, changing the phase angle from $90^{\circ}$ to $+90^{\circ}$ as shown in Figure $3 \mathrm{~b}$.

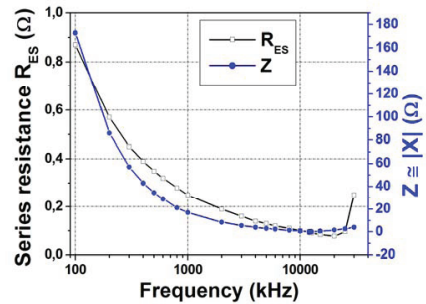

(a)

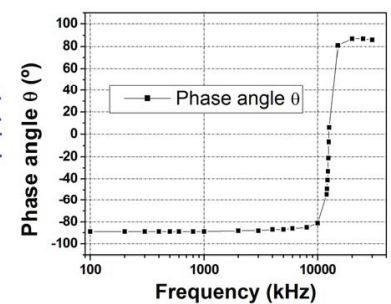

(b)
Figure 3. Frequency response for ceramic capacitor of $10 \mathrm{nF}$ showing: (a) $\mathrm{Z}$ and $\mathrm{R}_{\mathrm{ES}}$ and (b) phase angle.

Figure 4a gives the corresponding capacitance variation (in black) with frequency, in which it is observed that the capacitance is basically stable up to approximately $10 \mathrm{MHz}$ with sudden capacitance increase basically caused by the resonance between the capacitance and inductance of the capacitor leads at this frequency. Over this frequency the inductive effect is predominant, as shown in Figure 4a by the $\mathrm{L}$ curve in blue. Due to the increase of the current density during resonance, the charge accumulation occurs in a very short time, increasing the capacitance and, consequently, losses, as indicated by the peak in $\tan \delta$ curve shown in Figure 4b. In any event, the losses (or $\tan \delta$ ) tend to increase as frequency increases because of a decrease in the parallel dielectric resistance $\mathrm{R}_{\mathrm{D}}$ with $\mathrm{f}$ [8].

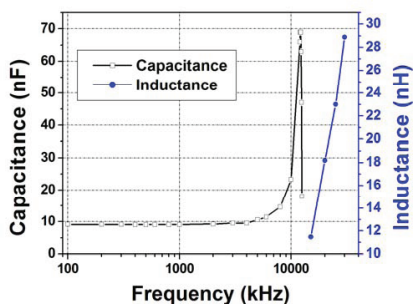

(a)

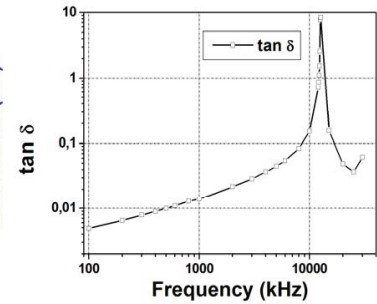

(b)
Figure 4. Capacitance (a) and tangent loss (b) measured for the $10 \mathrm{nF}$ capacitor as a function of frequency.

A similar behavior for dielectric response as a function of frequency was observed for type 4 and 8 PZT samples because of the device terminal inductance. As an illustration, Figure 5a shows the capacitance variation as a function of frequency for the type 8 PZT sample, including the inductive phase, and Figure $5 \mathrm{~b}$ gives the corresponding loss tangent with a peak near $5 \mathrm{MHz}$.

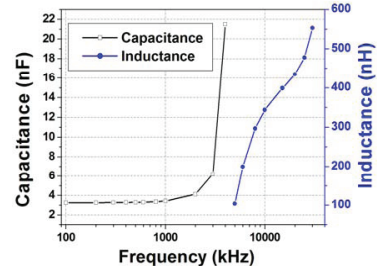

(a)

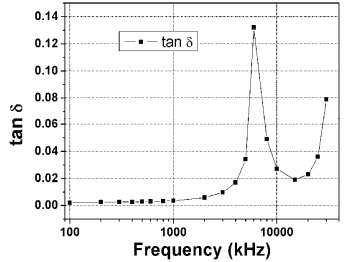

(b)
Figure 5. Capacitance (a) and loss tangent (b) measured for the type 8 PZT as a function of frequency.

In short, the main point in the frequency response characterization is the comparison of losses between dielectrics since a lower dissipation factor leads to a better dielectric. This is particularly important for applications in capacitive NLTLs, where high losses have been a limiting factor for high frequency operation $[1,2]$. Figure 6 gives the measured loss tangent of de-poled PZTs (type 4 and 8 ) and of several capacitors up to $2 \mathrm{MHz}$ below the resonant frequency of the dielectrics, where it is verified that PZT materials have a better performance than BST dielectrics in this frequency range. The reason is that their dielectric resistance $R_{D}$ is significantly higher as dissipation factor $\mathrm{D}$ in this case is given by $\tan \delta=1 /\left(\omega \times C \times R_{D}\right)$. Figure 7 illustrates this by showing a comparison between dielectric resistances from PZT (type 8) and BST (1 $\mathrm{nF}$ capacitor) calculated from D [8].

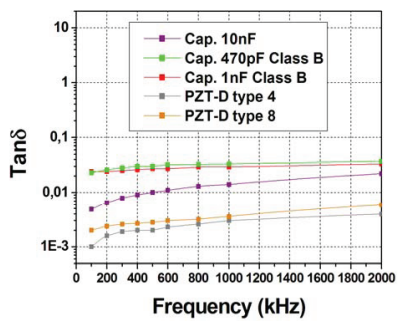

Figure 6. Loss tangent comparison shown between several dielectrics (PZTs and capacitor BSTs)

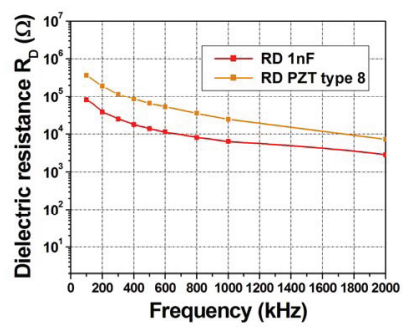

Figure 7. Comparison between PZT and BST dielectric resistances calculated from loss tangent.

The lower dissipation factor observed for de-poled PZTs (at least at lower frequencies) is also confirmed through $\mathrm{P} \times \mathrm{E}$ polarization measurements using $60 \mathrm{~Hz}$ alternating fields [5]. The bigger area inside the $\mathrm{P} \times \mathrm{E}$ curve means higher losses. For instance, the $\mathrm{P} \times \mathrm{E}$ curve measured of capacitor of $1 \mathrm{nF} / 2 \mathrm{kV}$ shows higher losses than that of de-poled type 8 PZT as shown in Figures $8 \mathrm{a}$ and $8 \mathrm{~b}$, respectively. 


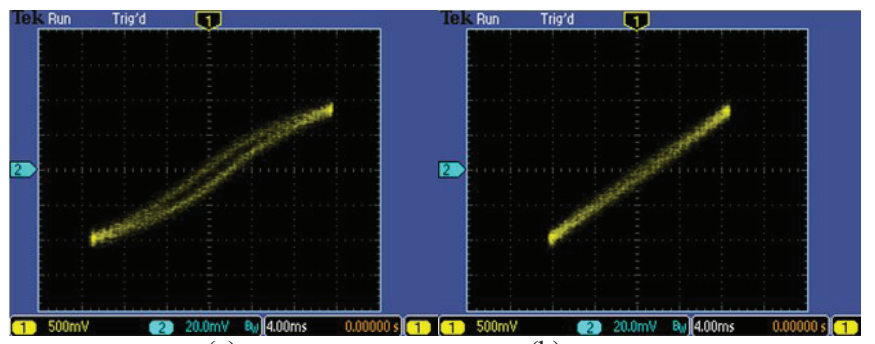

(a)

(b)

Figure 8. P $\times$ E loops measured for: (a) capacitor of $1 \mathrm{nF} / 2 \mathrm{kV}$ and (b) depoled type 8 PZT.

On the other hand, as expected, poled PZT presented higher losses than de-poled ones, as illustrated by their polarization curves given in Figures 9a and 9b, respectively. Observe that the poled PZT in Figure 9a presented higher losses because of the bigger area of its internal $\mathrm{P} \times \mathrm{E}$ loop.

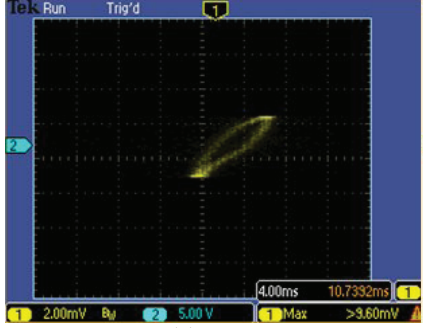

(a)

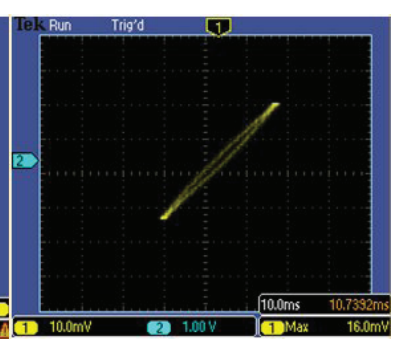

(b)
Figure 9. $\mathrm{P} \times \mathrm{E}$ loops measured for comparison between a poled type 4 PZT (a) and a de-poled type 4 PZT (b).

For the BD tests, the capacitor terminals were directly connected to the HV pulser output while a second sample holder with smaller electrode area $(<5 \mathrm{~mm}$ in diameter) was especially built for PZT-pulser connection to provide lower sample capacitances $(\leq 1 \mathrm{nF})$. For this, the PZT ceramic silver coatings were completely removed by polishing both surface sides with thin sand paper. A lower capacitance on HV BD tests is desired because it causes much less attenuation on the primary voltage, allowing higher output pulse amplitudes during $\mathrm{BD}$. The Weibull (WB) plots [3] obtained for 10 samples of each device (PZT-4 samples and ceramic capacitors of $0.47 \mathrm{nF} / 3 \mathrm{kV}$ ) are shown in Figures 10a and $10 \mathrm{~b}$, respectively. From Figure 10a, an average BD strength of about $4.37 \mathrm{kV} / \mathrm{mm}$ for type 4 PZT with a standard deviation of $\pm 0.36 \mathrm{kV} / \mathrm{mm}$ was obtained. Similarly, from Figure $10 \mathrm{~b}$, an average BD strength on the order of $8.72 \mathrm{kV} / \mathrm{mm}$ was obtained for the ceramic capacitor of $0.47 \mathrm{nF} / 3 \mathrm{kV}$ with a standard deviation of $\pm 0.81 \mathrm{kV} / \mathrm{mm}$. Comparing with BST capacitors, these results show that PZTs have approximately half the pulsed BD strength of ceramic capacitors.

\section{CONCLUSION}

De-poled PZT ceramics have been studied in terms of their nonlinear properties and dielectric breakdown strength. The acquired PZT samples had their piezoelectric properties removed by thermal de-poling at the factory to limit dielectric hysteresis losses at high frequencies, which was confirmed as expected by the material polarization curve $\mathrm{P} \times \mathrm{E}$ measured with negligible internal area. The results obtained herein so far indicate that de-poled PZTs have lower dielectric constant (about 1000) and lower losses (dissipation factor D in the range of 0.01 up to $2 \mathrm{MHz}$ ) compared to BST dielectrics used in commercial capacitors (5000 and 0.1 range, respectively), whose use appears to be promising for NLCL high frequency operation although they are less nonlinear $(17 \%)$ and present lower breakdown strength $(\approx 5 \mathrm{kV} / \mathrm{mm})$ than BSTs $(50 \%$ and $\approx 10 \mathrm{kV} / \mathrm{mm}$, respectively). In this way, we expect the frequency operation of NLCLs may be extended above 100$250 \mathrm{MHz}$ as amplitudes of the output oscillating pulses would not be damped hugely by the dielectric losses as the quality factor is given by $\mathrm{Q}=1 / \mathrm{D}$ [1]. Therefore, the project's next steps will be: a) to extend dielectric $\tan \delta$ measurements up to $1 \mathrm{GHz}$, and b) to test a NLCL built with de-poled PZTs to verify the prospects for a high frequency better performance.

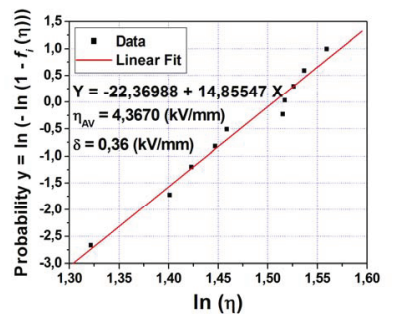

(a)

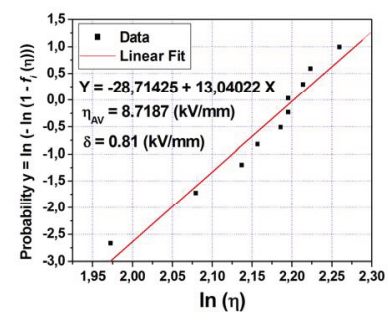

(b)
Figure 10. WB plots for: (a) type 4 de-poled PZT and (b) $0.47 \mathrm{nF} / 3 \mathrm{kV}$ capacitor, being y the probability and $\eta$ the BD strength in $\mathrm{kV} / \mathrm{mm}$.

\section{ACKNOWLEDGMENT}

Authors acknowledge USAF-SOARD for their funding support under contract number no. FA9550-10-1-0013.

\section{REFERENCES}

[1] ] P. W. Smith, "Pulsed, high Power, RF generation from nonlinear dielectric ladder networks - performance limits," in Proceedings of the 18th IEEE Int. Pulsed Power Conf., Chicago, IL, pp. 167- 172, 2011.

[2] H. Ikezi, J.S. DeGrassie, and J. Drake, "Soliton generation at $10 \mathrm{MW}$ level in the very high frequency band," Appl. Phys. Lett., vol. 58, no. 9, pp. 986-987, March 1991.

[3] P.A. Castro. Studies of electric breakdown under pulsed conditions. 71p. thesis-Msc. (In Electrical Engineering- Electrical and Computer Eng. Dept.) - University of New Mexico (UNM), NM, May 2010.

[4] J.O. Rossi, L.P. Silva Neto, and A.R Silva Jr., "Study of HV dielectric Ceramics for applications in compact pulsed power" in Proc. of the 18th IEEE Int. Pulsed Power Conference, Chicago, IL, USA, pp. 459-464, 2011.

[5] J.C. Burfoot and G. W. Taylor. Polar dielectric and their applications. Los Angeles: University of California Press, p. 465, 1979.

[6] S. Roberts, "Dielectric and piezoelectric properties of barium titanate," Physical Review, vol. 71, no. 12, pp. 890-895, 1947.

[7] K. M. Johnson, "Variation of dielectric constant with voltage in ferroelectrics and its application to parametric devices," J. Applied Phys., vol. 33, no. 9, September 1962, pp. 2826-2831.

[8] QuadTech. Equivalent series resistance (ESR) of capacitors. Application Note. July 2003, http://www.quadetch.com. 